\title{
Şönt Reaktör Geçici Zaman Analizi ve Üşüşsme Akımı Hesaplaması için Matlab Tabanlı Yazılım Geliştirilmesi
}

\author{
İsmail TOPALOĞLU*1

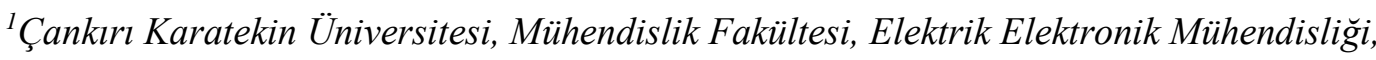 \\ Çankırı
}

Geliş tarihi: 26.01.2016 Kabul tarihi: 09.05.2016

\section{Özet}

Şönt reaktörler yüksek gerilim hatlarında yük değişimlerinden oluşan gerilim dalgalanmalarını önlemek ve kapasitif yükü absorbe etmek için kullanılmaktadır. Şönt reaktörler enerji iletim hatlarında sürekli devrede kalabildikleri gibi yük durumuna göre de devreye alınabilmektedir. Şönt reaktörler devreye girerken geçici olaylar oluşmaktadır ve üşüşme akımı da bunlardan en önemlisidir. Bu çalışmada şönt reaktörün geçici zaman analizi için sensör tabanlı bir metot önerilmektedir. Bu ölçüm için bilgisayar tabanlı bir yazılım geliştirilmiştir. Geliştirilen ara yüz sayesinde temel sistem parametreleri sistem gerilimi, çalışma frekansı, birleştirme kapasite değerleri, 3 fazlı sargı bağlantı hesapları, şebeke kısa devre gücü ve sistem toplam empedans değerleri girilebilmektedir. Önerilen ölçüm sisteminde yer alan sensörlerden elde edilen bilgiden sistem kapasite değerleri elde edilebilmektedir. Geliştirilen sistemin en önemli özelliği şönt reaktör devreye alındığında oluşacak akım ve gerilim değerlerinin analizine imkân veriyor olmasidır.

Anahtar Kelimeler: Şönt reaktör, Geçici zaman analizi, Üşüşme akımı

\section{Development of Matlab Based Analysis Software for Transient Analysis of Shunt Reactor and Inrush Current Calculation}

\begin{abstract}
Shunt reactors to prevent voltage fluctuations that occur from changes in load and capacitive load of highvoltage lines are used to absorb. Shunt reactors at power transmission lines according to the load condition remains active as they can be switched on continuously. Shunt reactors are composed transients entering the circuit and inrush current is the most important of them. In this study, sensor-based methods

Yazışmaların yapılacağı yazar: İsmail TOPALOĞLU, Çankırı Karatekin Üniversitesi, Elektrik Elektronik Mühendisliği, Çankırı, itopaloglu@karatekin.edu.tr
\end{abstract}


for transient analysis of shunt reactors are recommended. Computer-based software developed for this measurement. This newly developed interface basic system parameters, system voltage, operating frequency, capacitance values merge, 3-phase winding up accounts; network and system total power short circuit impedance values can be entered. Recommended information from the system capacity values obtained from sensors located in the measurement system can be obtained. The most important feature is that the developed system allows the analysis of current and shunt reactors will occur when the voltage is switched on.

Keywords: Shunt reactor, Transient analysis, Inrush current

\section{GíRiș}

Şönt reaktörlerin enerji iletim hatlarında kullanım amaçları gerilim yükselmesini azaltmaktır. Enerji iletim hatlarında yer altı kablolarının oluşturduğu aşırı kapasitif yükleri bastırmak için şönt reaktörler kısmen veya hep beraber devreye alınabilirler. Bu sayede enerji iletim hattına ekstra reaktif güç sağlayabilirler. Ancak yüksek gerilim hatlarında şönt reaktörler devreye alınırken güç sistemi üzerinde geçici olaylar meydana getirirler. Son dönemlerde çok yüksek gerilim hatlarındaki hızlı gelişmeler şönt reaktörün dinamik kapasitif güç kompanzasyonu için kullanımını zorunlu hale getirmiştir. Yapılan birçok araştırma çalışmasında şönt reaktörün ayırıcılar üzerindeki geçici olayları incelenmiştir [1-5]. Şönt reaktörün kontrol edilebilirliği, çalışma karakteristikleri ve farklı bağlantı şekillerinin etkileri incelenmiştir [6-10]. Geleneksel güç kompanzasyon araçları alçak gerilim şönt kapasitör gurupları, statik VAR kompanzatörleri ve jeneratör gurupları istenilen reaktif güç regülasyonunu ve aşırı gerilim yükselmesini sağlayamamaktadırlar [11-13]. Şönt reaktöre ait üşüşme akımı ve geçici olaylar örnek uygulamalar ile birçok çalışmada incelenmiştir [14]. Bu çalışmada Matlab GUI tabanlı bir yazılım ile dişarıdan şönt reaktörün bağlı bulunduğu sistemin parametreleri girilmekte ve sensör tabanlı bir ölçüm ile sistemde oluşan geçici olaylar analiz edilip hem grafik hem de sayısal sonuç olarak gösterilebilmektedir. $\mathrm{Bu}$ durum çalışmanın en önemli kazanımı olarak söylenebilir. Dahası yazılıma sonradan eklenebilecek eş zamanlı veri işleme kabiliyeti ile otomatik olarak sistemin geçici zaman analizi ve üşüşme akımı hesaplamaları yapılabilecektir.

\section{2. ŞÖNT REAKTÖR GEÇICİ ZAMAN ANALIZi}

Şönt reaktör geçici zaman analizinde temel alınan eşdeğer devrede seri sarg1 direnci, sarg1 indüktansı ve enerji hattı direnci ve indüktansı hesaba katılmıştır. Eşitlik1, 2, 3 ve 4 temel devre denklemleridir.

$$
\begin{aligned}
& e=i_{L} R+u_{L} \Rightarrow i_{L}=\frac{e-u_{L}}{R} \\
& u_{L}=L \frac{d i_{L}}{d t} \\
& i_{L}=\frac{e-L \frac{d i_{L}}{d t}}{R} \Rightarrow i_{L}^{\prime}+\frac{R}{L} i_{L}-\frac{e}{L}=0 \\
& e=E_{\max } \operatorname{Sin}(\omega t+\alpha)
\end{aligned}
$$

Eşitlik 5 de zamana bağlı akımın enerji hattına bağlı olduğu durumda ayırıcı açıkken ve kapalıyken ki durumları ifade edilmiştir. Burada görüldüğü üzere anahtar açıkken şönt reaktör devrede değildir ve geçici durum değerleri ve kalıcı durum değerleri sıfırdır.

$$
\begin{aligned}
& i_{L}=i_{\text {Transient }}+i_{\text {Steady }} \\
& i_{\text {Transient }}+\left.i_{\text {Steady }}\right|_{t=0}=0, \\
& i_{\text {Transient }}+\left.i_{\text {Steady }}\right|_{t=\infty}=i_{\text {Steady }} \\
& |Z|=\sqrt{R^{2}+(\omega L)^{2}}
\end{aligned}
$$




$$
\begin{aligned}
& \arctan \left(\varphi_{U}-\varphi_{Z}\right) \cong \arctan \left(-\varphi_{Z}\right) \\
& =-\frac{\omega L}{R}\left\{\varphi_{Z}=0, \varphi_{Z}=\pi, \varphi_{Z}=2 \pi, K\right\}
\end{aligned}
$$

Eşitlik 6 ve 7 de devre empedansı ve faz açısı hesaplamaları verilmiştir.

$$
\begin{aligned}
& i_{L}(t)=i_{\text {Transient }}+i_{\text {Steady }}= \\
& A \cdot e^{-\frac{R}{L} t s}+\frac{e}{\sqrt{R^{2}+(2 \pi f L)^{2}}} \sin \left(2 \cdot \pi \cdot t s+\varphi_{U}-\varphi_{Z}\right) \\
& A=-\frac{e}{\sqrt{R^{2}+(2 \pi f L)^{2}}} \sin \left(2 \cdot \pi \cdot t s+\varphi_{U}-\varphi_{Z}\right) e^{-\frac{R}{L} t s}
\end{aligned}
$$

Eşitlik 8 ve 9 da kararlı durum ve geçici durum akım eşitlikleri verilmiştir. $\mathrm{Bu}$ eşitliklerde $\mathrm{A}$ katsayısı eşitlik 9 ile verilmiştir. Şönt reaktörün ayırıcı kapalı ve açıkken oluşan akım ve gerilim düşümü eşitlikleri sırasıyla eşitlik 10, 11, 12 ve 13 de verilmiştir.

Anahtar kapalı iken;

$$
i_{L}(t)=\left\{\begin{array}{l}
-\frac{e}{\sqrt{R^{2}+(2 \pi f L)^{2}}} \sin \left(2 \cdot \pi \cdot t s+\varphi_{U}-\varphi_{Z}\right) e^{-\frac{R}{L} t-t s} \\
+\frac{e}{\sqrt{R^{2}+(2 \pi f L)^{2}}} \sin \left(2 \cdot \pi \cdot t+\varphi_{U}-\varphi_{Z}\right)
\end{array}\right\}
$$

$$
u_{L}=\left\{\begin{array}{l}
-R \cdot \frac{e}{\sqrt{R^{2}+(2 \pi f L)^{2}}} \sin \left(2 \cdot \pi \cdot t s+\varphi_{U}-\varphi_{Z}\right) e^{-\frac{R}{L} t-t s} \\
+2 \pi f L \frac{e}{\sqrt{R^{2}+(2 \pi f L)^{2}}} \cos \left(2 \cdot \pi \cdot t+\varphi_{U}-\varphi_{Z}\right)
\end{array}\right\}
$$

Anahtar açıkken;

$$
i_{L}(t)=\left\{-\frac{e}{\sqrt{R^{2}+(2 \pi f)^{2}}} \sin \left(2 \cdot \pi \cdot t s+\varphi_{U}-\varphi_{Z}\right) e^{-\frac{R}{L} t-t s}\right\}
$$

$$
u_{L}=L \frac{d i_{L}}{d t}=\left\{\begin{array}{l}
-R \cdot \frac{e}{\sqrt{R^{2}+(2 \pi f L)^{2}}} \\
\cdot \sin \left(2 \cdot \pi \cdot t s+\varphi_{U}-\varphi_{Z}\right) e^{-\frac{R}{L} t-t s}
\end{array}\right\}
$$

\section{3. ŞÖNT REAKTÖR GEÇİCI ZAMAN ve ÜŞÜŞME AKIMI HESAPLAMA PROGRAMI}

Şönt reaktörler için temel yapılarını hesaba katan, üşüşme akımı ve geçici zaman analizi yapabilen Matlab GUI tabanlı bir yazılım geliştirilmiştir. Hem endüstride hem de akademide farklı yazılım tasarım programları kullanılmaktadır ancak hiçbirisi matlab matematik altyapısını sunmamaktadır. Geliştirilen analiz programı ile gerçek zamanlı sensör tabanlı analizler yapılabildiği gibi dışardan analiz başlangıç parametreleri girilerek de sonuçlar elde edilebilmektedir. Analiz programının düzgün çalışabilmesi için başlangıç analiz değerlerinin dışarıdan girilmesi gerekmektedir. Program çıktısı olarak;

a) Nümerik sayısal sonuçlar,

b) Faz gerilim grafikleri,

c) Üşüşme akımı grafikleri,

değerleri görüntülenebilmektedir. Analize başlamadan önce sargı bağlantı tipinin seçilmesi gereklidir. Program bu seçimden sonra girilen başlangıç analiz parametre değerlerini kullanarak empedans, üşüşme akımı, faz gerilimi etkin değerleri, hat kapasitansları, mesafe ve kapasitenin gerilim değerlerine etkisini hesaplamakta ve görsel olarak sonuç verebilmektedir. Hali hazırda analiz parametreleri dışarıdan yazılıma girilmekte saha çalışmaları analizi yapılabilmektedir. Çalışmanın bir sonraki versiyonu için otomatik saha değerlerini alıp analiz edebilme yeteneği kazandırılması hedeflenmektedir.

Şekil 1'de şönt reaktör geçici zaman analiz programı genel görüntüsü verilmiştir. Program uluslararası kullanılabilirliği olsun diye İngilizce hazırlanmıştır. 


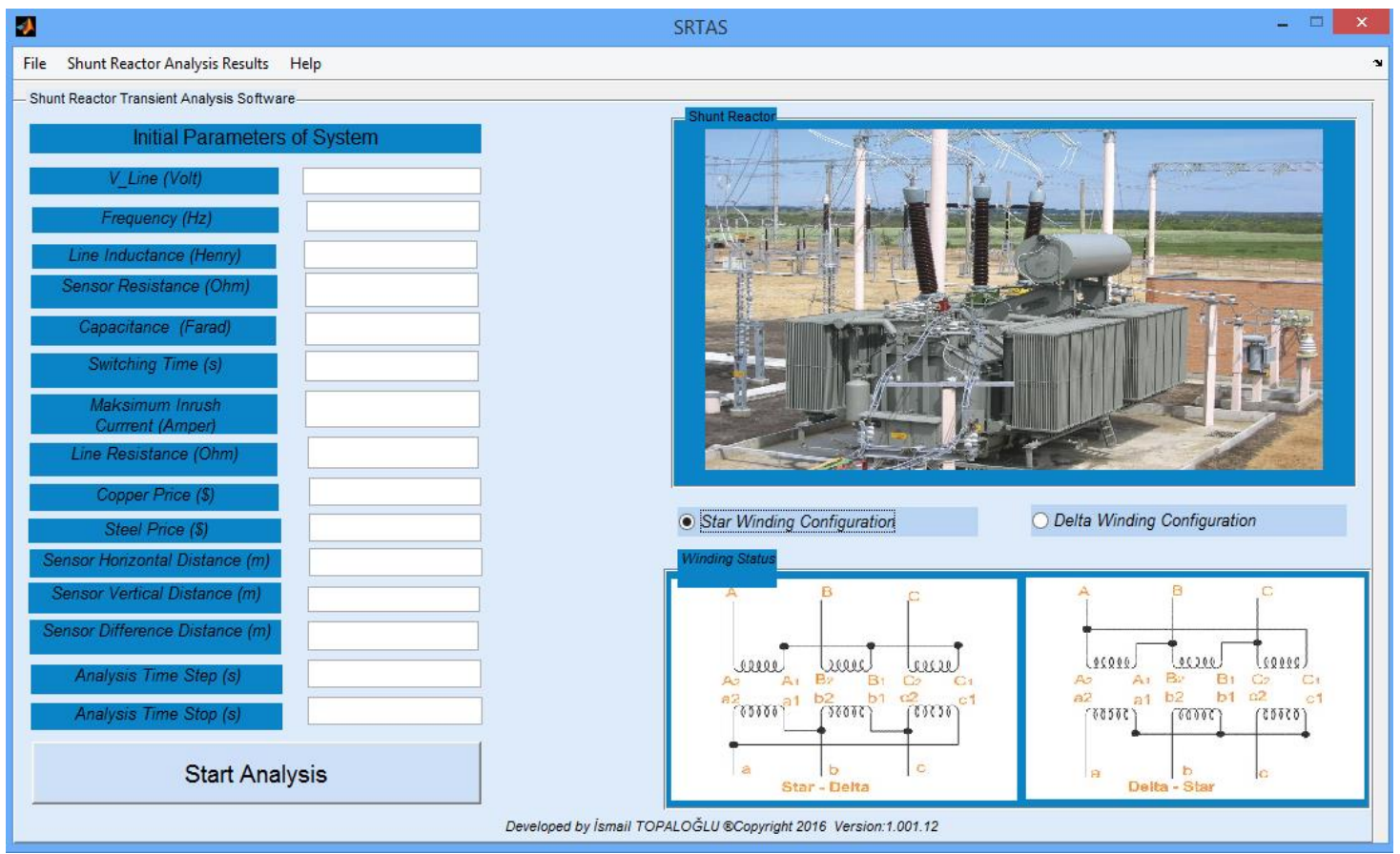

Şekil 1. Şönt reaktör geçici zaman analiz programı

\section{ANALIZ SONUÇLARI}

Program analiz sonuçlarını incelediğimiz zaman A, $B$ ve $C$ fazlarına ait dalga formlarının etkin değerlerinden oluşmuş üç faz sistem gerilim grafiği, sensör gerilim değerlerini ve sensör ölçümlerine göre yeniden oluşturulmuş gerilim dalga formları ve değerlerini inceleyebiliriz. Şekil 4'de görüldüğü üzere üç faz için üç sensör altyapısı kullanılmaktadır. Hali hazırda programda sensörler aktif olarak ölçüm yapmamaktadır. Ancak kullanılacak olan sensörün bilgileri girilerek, sensör direnci, kapasite değeri vb. gibi analizler yapılabilmektedir. Şekil 2'de görüldüğü üzere program sayısal tek sonuçları ayrı bir ekranda göstermeye imkân tanımaktadır. Buna ek olarak üşüşme akımına karşılık empedans ve faz gerilimlerini grafik olarak da gösterebilmektedir. Şönt reaktör geçici zaman analizini programın kolaylaştırması yanında devreye alındığı anda oluşan üşüşme akımını ayrı ayrı hesaplayıp hem grafik hem de sayısal sonuç olarak gösterebilmesi tasarlanan programın üstün yönlerindedir.

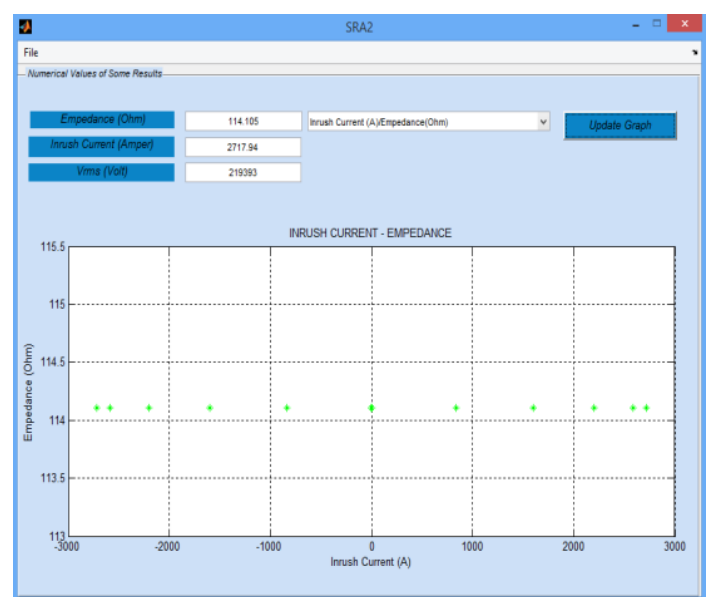

Şekil 2. Sayısal sonuçlar görünümü

Şekil 3'de üşüşme akımı ile hat kapasitanslarının etkisini içeren gerilim analiz sonuçları değerleri gösterilmektedir. Geliştirilen yazılım ile etkin ve uzaktan ölçüm ile bu değerler çok yüksek gerilim hatlarında elde edilebilmesine olanak sağlamaktadır. Ayrıca yazılım sonraki aşamada iteratif olarak şönt reaktör tasarımı yapabilir hale getirilecektir. 


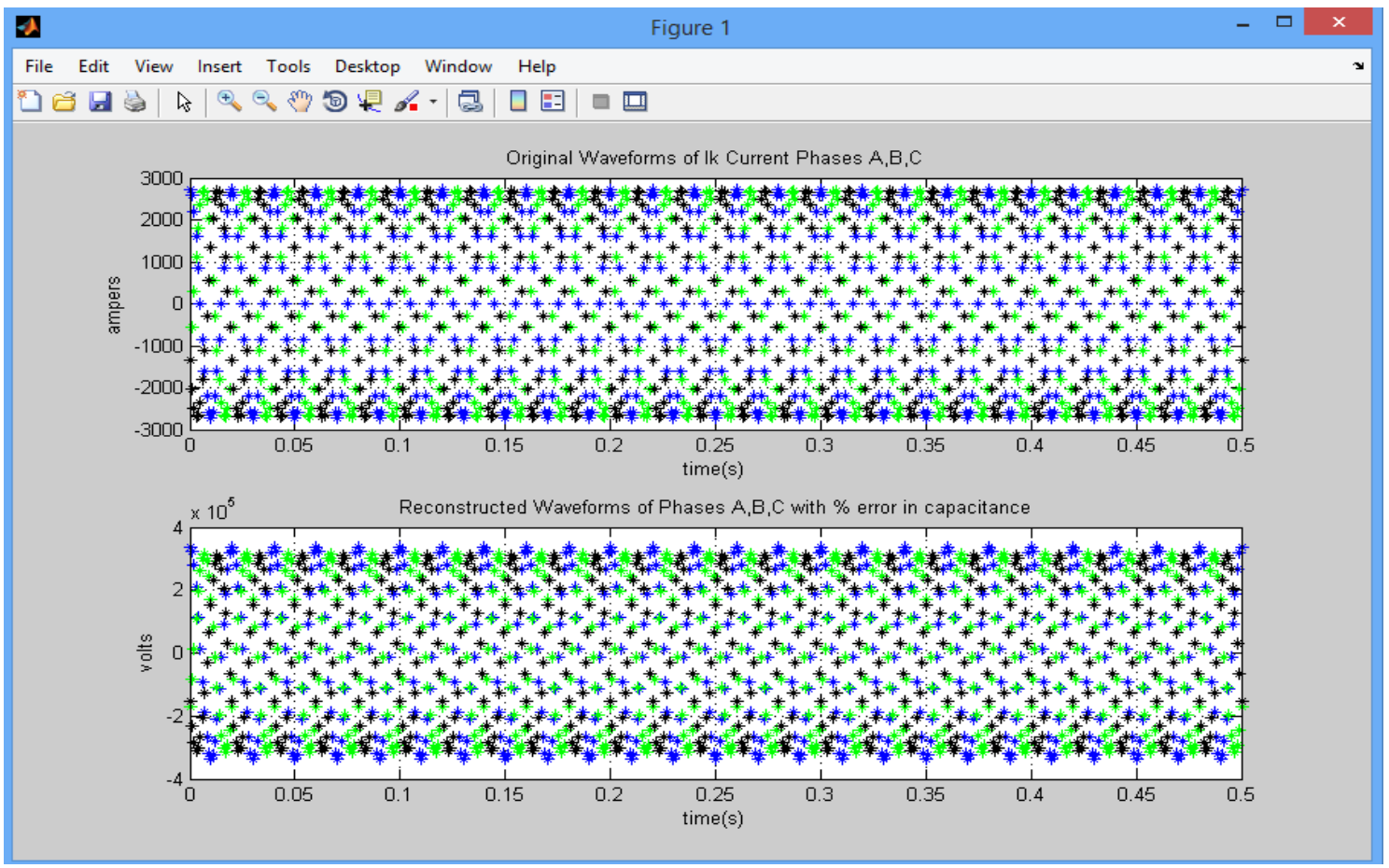

Şekil 3. Üşüşme akımı ve kapasitans etkili gerilim dalga şekilleri

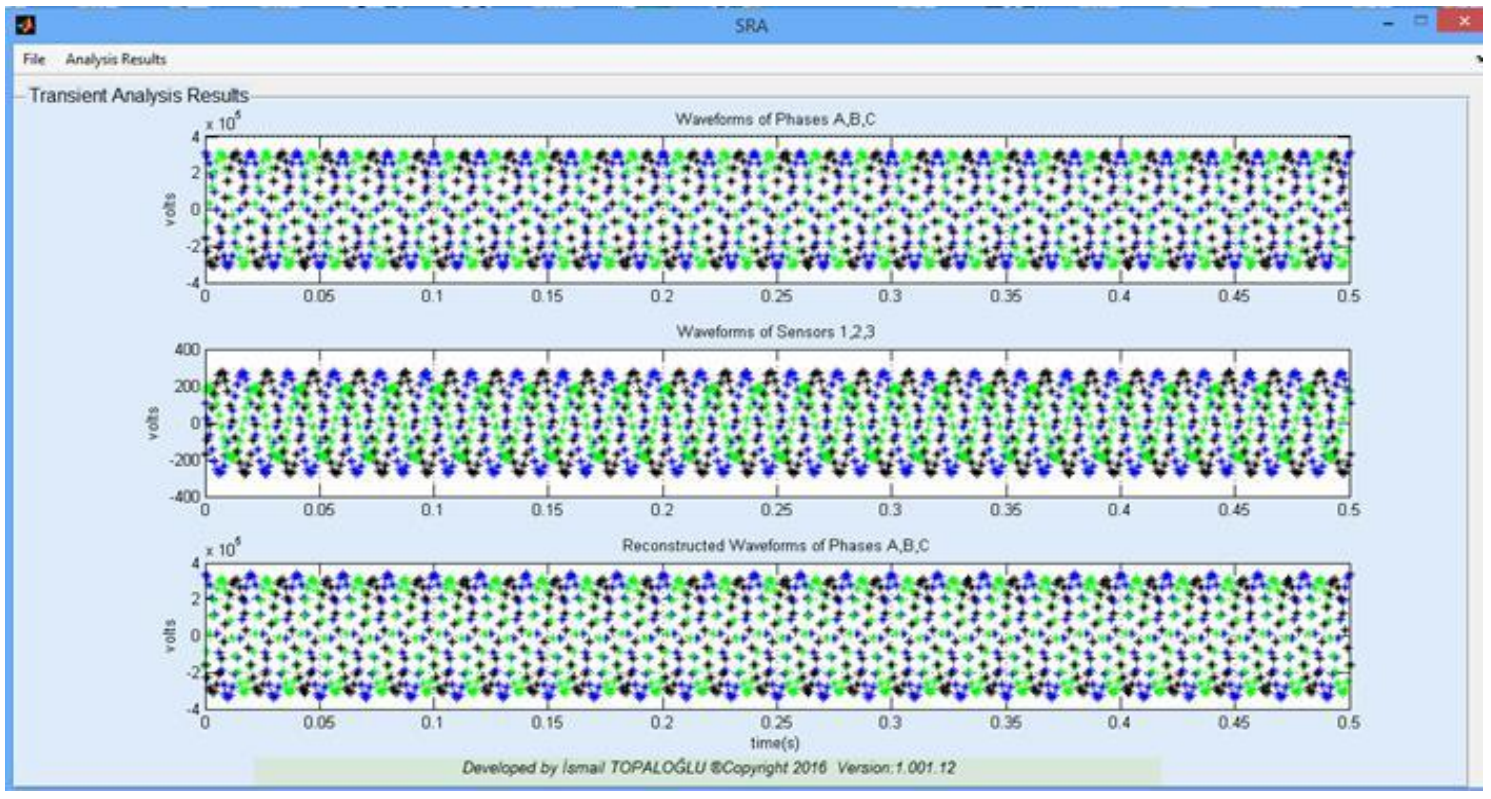

Şekil 4. Analiz gerilim dalga formları 
Çizelge 1'de örnek uygulamada kullanılan giriş analiz parametreleri değerleri verilmiştir. Analiz $380 \mathrm{kV}$ bir enerji iletim hattında kullanılan şönt reaktör değerlerinden elde edilmiştir.

Çizelge 1. Giriş analiz parametre değerleri

\begin{tabular}{|l|c|c|c|}
\hline \multicolumn{1}{|c|}{ Parametre Ad1 } & Simge & Birim & Değeri \\
\hline Çalışma gerilimi & $\mathrm{V}$ & $(\mathrm{V})$ & 380,000 \\
\hline Frekans & $\mathrm{f}$ & $(\mathrm{Hz})$ & 50 \\
\hline İndüktans & $\mathrm{L}$ & $(\mathrm{H})$ & 0,34 \\
\hline $\begin{array}{l}\text { Anahtarlama } \\
\text { zamanı }\end{array}$ & $\mathrm{t}$ & $(\mathrm{sn})$ & 0,25 \\
\hline Yatay uzunluk & $\mathrm{lg}$ & $(\mathrm{m})$ & 3 \\
\hline Sensör direnci & $\mathrm{Rs}$ & $\mathrm{M} \Omega$ & 6 \\
\hline Dikey uzunluk & $\mathrm{lh}$ & $(\mathrm{m})$ & 2 \\
\hline Kapasitans & $\mathrm{C}$ & $(\mathrm{pF})$ & 12 \\
\hline
\end{tabular}

\section{SONUÇLAR}

$\mathrm{Bu}$ çalışmada matlab tabanlı şönt reaktör geçici zaman analizi ve üşüşme akımı analizlerinde kullanılabilecek bir yazılım geliştirilmiş ve örnek uygulama yapılmıştır. Geliştirilen yazılım sayesinde çok yüksek gerilim hatlarında şönt reaktör devreye alındığ 1 anda oluşan gerilim ve üşüşme akımı hem sayısal hem de grafik sonucu olarak gösterilebilmektedir. Geliştirilen program enerji iletim hattı kapasitans değerleri ve bunların etkisini de hesaplamalara dâhil etmektedir. Bunun yanı sıra sensör tabanlı ölçüm yapabilme kabiliyeti vardır. Bundan sonraki çalışmada yazılımın şönt reaktör tasarımı yapabilir hale getirilmesi hedeflenmektedir. $\mathrm{Bu}$ kabiliyetlerinin yanında yazılım üretim maliyeti ve işletme maliyeti analizlerini de yapabilecektir.

\section{KAYNAKLAR}

1. Bossche, V., Cekov, A., Vencislav, V., 2005. Inductors and Transformers for Power Electronics. Boca Raton, FL, USA: Taylor \& Francis Group
2. All Circuit Models were Made in Tina for Windows-the Complete Electronics Lab. Software Made by DesignSoft: http:// www.designsoftware.com

3. Three-Phase Line Reactor Model was Adapted from Online MATLAB Library - 3-Winding 3Phase Transformer Model: http://www.kxcad.net/cae_MATLAB/toolbox/p hysmod/powersys/ref/threephasetransformerind uctancematrixtypethreewindings.html

4. Quad Tech. LCR Primer Measurement Instruction Manual. Massachusetts USA. http:// www.quadtech.com

5. Ramli, M.S., 2008. Investigation of Circuit Breaker Switching Transients for Shunt Reactors and Shunt Capacitors, Master Thesis, Avustralya.

6. Chen Weixian, Chen He, Lu tiecheng, Zhou Wenjun, 2005. Ultra-high Voltage Controllable Shunt Reactor, High Voltage Eengineering, 31, (11), pp.26-27.

7. Hasibar R M, Legate A C,Brunke L, 1981. The Application of Highspeed Grounding Switches for Single-Pole Reclosing on 500kV Power Systems,. IEEE Trans on PAS, 100(4), pp:1512-1515.

8. Chen, B., Kokermak, J, M, Torrey, D, A, 1999. Harmonicfree Single-Phase Controlled Saturable Reactor Using Active Filtering, IEEE international Conference on Power Electronic and Drive System, PEDS'99, pp.1037-1042.

9. Zhou, Q, Guo, Q, Guangquan B., Liangeng B., 2007. Application of Controllable Reactors in China's Power Grid at Extra and Ultra Voltage Level, Proceeding of the CSEE, 27(7), pp.1-6.

10. Tian, C, Chen, B, 2005. Application of Controlled Reactor in Northwest 750kV Power System, in High Voltage Engineering, 31(3), pp.18-21.

11. Xu, W, Chen, S, Jinliang H., 2005. Research on Switching Over-Voltage Caused by Faults in $1000 \mathrm{kV}$ UHV ac transmission line, Power System Technology, 29(21), pp.10-13.

12. Chen, H, Chen, W., 2002. Choice of Inductance on Neutral of Shunt Reactors,"High Voltage Engineering, 28(8), pp.9-10.

13.Zhou, L., Zhu, Y., Zhou, Z, 2006, Regulating Range of UHV Controlled Shunt Reactor, 
Transactions of China Electro Technical Society, 21(12) pp.116-120.

14.Zhang, L., Xu, Y., 2007, Application and Devdlopment of Shunt Reactors in EHV \& UHV Transmission Lines, Electric Power Automation Equipment, 27(4), pp.75-78. 
